Canadian Oncology

Nursing Journal

Revue canadienne

de soins infirmiers

en oncologie

Volume 31, Issue 2 • Spring 2021

elSSN: 2368-8076 


\title{
Infirmière de recherche clinique en oncologie : revue exploratoire
}

\author{
par Mai N. Hong, K. Alix Hayden, Shelley Raffin Bouchal et Shane Sinclair
}

\section{RÉSUMÉ}

En ce $21^{e}$ siècle, le cancer est une maladie qui suscite particulièrement l'attention en raison de sa complexité ainsi que des impacts physiques, émotionnels et financiers sur notre vie. L'attention portée à la recherche et les investissements dans le traitement contre le cancer en font la maladie la plus étudiée dans les essais cliniques à l'échelle mondiale. Les infirmières de recherche clinique font partie de l'équipe de recherche en oncologie et sont un élément fondamental de réussite des essais. Leurs relations directes avec les participants d'une étude de recherche sont essentielles pour les activités des essais cliniques en première ligne. De façon générale, l'afflux et la complexité des essais cliniques en oncologie ont transformé la pratique infirmière en oncologie et ont mené à la création de la sous-spécialité unique qu'est linfirmière de recherche clinique en oncologie. La présente revue exploratoire s'est penchée sur le rôle et la pratique futurs de linfirmière de recherche clinique.

Mots-clés : oncologie, essais cliniques, infirmière de recherche clinique, soins infirmiers

\section{CONTEXTE}

$\mathrm{L}$ e rôle d'infirmière de recherche clinique a fait son apparition dans les années 1950, et a passablement évolué depuis, passant par différentes nomenclatures (Jenkens et Hubbard,

\section{AUTEURS}

Mai N. Hong, B.Sc.inf., BN, M.S.inf., Étudiante diplômée, Faculté des sciences infirmières, Université de Calgary, 2500 University Drive NW, Calgary (Alberta), Canada T2N 1N4

K. Alix Hayden, MSBI, M.Sc., Ph.D., bibliothécaire, Libraries \& Cultural Resources, Université de Calgary, Taylor Family Digital Library, 2500 University Drive NW, Calgary (Alberta) T2N $1 \mathrm{~N} 4$

Téléphone : 403-220-3752

Shelley Raffin Bouchal, Ph.D., professeure agrégée, Faculté des sciences infirmières, Université de Calgary, 2500 University Drive NW Calgary (Alberta), Canada T2N 1N4

Téléphone : 403-220-6258

Shane Sinclair, Ph.D., professeur agrégé, Faculté des sciences infirmières, Université de Calgary, 2500 University Drive NW, Calgary (Alberta), Canada T2N 1N4 ; Compassion Research Lab, Université de Calgary, 2500 University Drive NW Calgary (Alberta), Canada T2N 1N4 ; Department of Oncology, Cumming School of Medicine, Université de Calgary, 2500 University Drive NW, Calgary (Alberta) Canada T2N 1N4

Téléphone : 403-220-2925

Auteur-ressource : Shane Sinclair

Téléphone : 403-220-2925

Courriel : sinclair@ucalgary.ca

DOI:10.5737/23688076312150164
1991; Medical Research Council Investigation, 1948). Voici quelques titres utilisés pour décrire les fonctions de l'infirmière de recherche clinique : gestionnaire de données, infirmière en thérapies expérimentales, infirmière chercheuse, infirmière coordonnatrice de recherche et infirmière de recherche clinique (Carlson, Reilly et Hitchens, 2005; Coulson et Phelan, 2000; Loh, Butow, Brown et Boyle, 2002; Ness et Royce, 2017). Par rapport à l'infirmière coordonnatrice de recherche et à ces autres titres axés sur l'application du protocole, le rôle de l'infirmière de recherche clinique (IRC) englobe également les soins prodigués directement aux participants de l'étude (Ehrenberger et Lillington, 2004; Nagel, Gender et Bonner, 2010). Ces autres fonctions incombent également à l'IRC : spécialiste de l'éducation aux patients et aux familles, fournisseur de soins, défenseur des droits des patients, gestionnaire, chercheur (McEvoy, Cannon et MacDermott, 1991). (McEvoy, Cannon et MacDermott, 1991). De plus, certaines activités cliniques sont propres à la pratique de l'IRC : éducation des patients (notamment sur la gestion des symptômes et des médicaments), dépistage et confirmation de l'admissibilité, rôle de défendeur concernant l'inclusion du patient et ses droits pendant l'étude, assistance aux patients pour la prise de décision, obtention du consentement éclairé (Castro et al., 2011; Mueller, 2001). En termes de responsabilités liées à la recherche, l'IRC tient un registre précis des réponses du patient et des manifestations indésirables, aide à la collecte de données, et sert parfois de liaison entre les collègues des soins infirmiers, le personnel, les patients et l'équipe de recherche (Castro et al., 2011; Mueller, 2001). Aujourd'hui, on reconnaît que l'IRC joue un rôle particulièrement essentiel et représente une ressource en matière d'essais cliniques (Hastings, Fisher, McCabe et Consortium, 2012).

Le développement des soins infirmiers de recherche clinique continue d'évoluer, mais non sans difficultés. Ainsi, comme la composition d'une équipe de recherche clinique varie en ce qui a trait aux membres et aux professionnels de la santé participants ainsi qu'en raison de la taille et du budget de l'étude de recherche, les rôles et les responsabilités de chacun peuvent évoluer et prendre de l'ampleur. Pour les infirmières œuvrant dans les établissements de recherche clinique, les rôles, les responsabilités, les titres et les descriptions de tâches semblent diversifiés. De plus, l'IRC et l'infirmière coordonnatrice de recherche peuvent aussi assumer des responsabilités qui se recoupent. Une telle diversité dans les rôles, les responsabilités et les titres d'emploi nécessitent une clarification des rôles, une définition de la portée et l'élaboration de cadres de pratique.

Pour reconnaître la pratique spécialisée des soins infirmiers de recherche clinique, un cadre conceptuel représentant la pratique de l'IRC a été élaboré, sur la base d'un projet 
de quatre ans mené de 2007 à 2011 (Castro et al., 2011; CRN, 2010 Domain of Practice Committee, 2009). Le cadre comprend ces cinq domaines de pratique : pratique clinique, gestion de l'étude, coordination et continuité des soins, protection des sujets humains, contribution à la science. Bevans et collaborateurs (2017) ont utilisé ce modèle pour clarifier le recoupement entre les deux rôles (IRC et infirmière coordonnatrice de recherche), en mentionnant que la pratique de l'IRC est fortement centrée sur la pratique clinique par rapport à la pratique de l'infirmière coordonnatrice de recherche, laquelle est largement axée sur la coordination de la recherche, la gestion de l'étude et la mise en œuvre du protocole. En réalité, bien qu'il s'agisse d'une distinction importante, de nombreuses infirmières sont embauchées pour assumer le double rôle d'IRC et d'infirmière coordonnatrice de recherche. En raison des demandes de services venant du secteur de la recherche clinique, la pratique de l'IRC continue d'augmenter et on a dû élaborer des lignes directrices pour la pratique professionnelle. Il s'agit désormais d'une spécialité reconnue des soins infirmiers en oncologie, comme en témoigne la fondation de l'International Association of Clinical Research Nurses (IACRN) en 2009, la reconnaissance en tant que spécialité de la pratique par l'American Nurses Association (ANA), et la rédaction en 2016 par l'ANA et l'IACRN d'un document exposant des normes de pratique et la portée des soins infirmiers dans le domaine des essais cliniques (Clinical Trial Nursing - Scope and Standards of Practice). Ce document reconnaît le cadre de pratique particulier des essais cliniques et de la recherche et me l'accent sur les compétences, les connaissances et l'éducation requises pour la prise en charge spécialisée des patients ou des sujets participant à des essais cliniques.

Les données actuelles de l'International Clinical Trials Registry Platform Search Portal (ICTRP) de l'Organisation mondiale de la Santé (OMS, 2017) indiquent que le Canada a actuellement 24789 essais cliniques, ce qui le classe au 7e rang dans le monde. Avec un total de 69745 essais à l'échelle planétaire, le cancer est la maladie la plus étudiée. Le nombre d'essais cliniques sur le cancer continue d'augmenter rapidement avec la multiplication des découvertes scientifiques et les technologies agiles dans l'élaboration des médicaments, accentuant la complexité des essais cliniques sur le cancer et la prise en charge des sujets d'étude. Cette intensification de ces problèmes de santé et augmentation des interventions médicales visant à les traiter viennent complexifier les soins aux patients et nécessitent des compétences spécialisées de la part des infirmières en oncologie, des circonstances opportunes pour un recours aux infirmières spécialisées (Peplau, 2003). Actuellement, en raison de la prévalence du cancer, la recherche sur cette maladie progresse et les essais cliniques se multiplient. Cette tendance accentue la demande d'IRC en oncologie et, par conséquent, les occasions de développement et d'expansion de son rôle. Les qualifications de l'IRC en oncologie, la normalisation de la pratique et la planification de la main-d'œuvre sont primordiales pour répondre au grand nombre d'essais cliniques sur le cancer et aux besoins de plus en plus complexes des patients cancéreux.
Étant donné que les soins infirmiers en recherche clinique constituent un nouveau domaine de pratique et que le nombre d'essais cliniques sur le cancer augmente sans cesse, cette étude a pour but d'examiner l'émergence et la pratique actuelle et future du rôle de l'IRC dans les établissements d'oncologie. La question ayant guidé l'étude a été : Que sait-on sur le rôle de l'IRC en oncologie?

\section{MÉTHODOLOGIE}

Ce projet a utilisé une méthodologie de revue exploratoire selon le cadre méthodologique proposé par Arksey et O’Malley (2005). Ce cadre compte six étapes : 1) Identification de la question de recherche; 2) Identification des études pertinentes; 3) Sélection des études; 4) Organisation des données; 5) Collecte, synthèse et communication des résultats; 6) Exercice de consultation facultatif. Les résultats sont présentés conformément à la Preferred Reporting Items for Systematic Reviews and Meta-Analysis Extension for Scoping Reviews (PRISMA-ScR) (annexe 1).

\section{Stratégie de recherches}

Trois membres de l'équipe de recherche $(\mathrm{MH}$, SS et $\mathrm{KAH}$ [bibliothécaire de recherche]) ont élaboré la stratégie de recherche pour identifier les études sur le rôle des IRC en oncologie. Le Cochrane Handbook précise que Cochrane Central Register of Controlled Trials (CENTRAL), Medline et Embase sont les principales bases de données pour les revues (Lefebvre et al., 2020). Aussi, dans le Cochrane, on suggère de chercher des bases de données spécifiques à la discipline et pertinentes à la question de recherche (Lefebvre et al., 2020). Sous la direction de la bibliothécaire de recherche, six bases de données pertinentes ont donc été sélectionnées pour la recherche, car elles indexent les revues sur les soins infirmiers, les sciences cliniques ainsi que le système de soins de santé et la pratique qui se rapportent à notre revue exploratrice. Les bases de données de la plateforme OVID comprenaient : Medline et les articles publiés avant impression (annexe 2), articles en cours, autres citations non indexées, mises à jour quotidiennes, EMBASE, PsycInfo, et le registre Cochrane des essais contrôlés. Les autres bases de données étaient CINAHL (Ebsco) et Scopus. Quatre concepts de recherche ont été ciblés : oncologie, essai clinique, infirmière en essais cliniques, infirmière de recherche clinique et soins infirmiers. Les recherches ont été effectuées à l'aide des mots-clés et des rubriques de chaque concept. La mise en correspondance des termes a été répétée dans chaque base de données, car les titres des bases de données variaient. Les mots-clés utilisés étaient les mêmes dans toutes les bases. La recherche a été menée entre janvier et mars 2018, puis répétée en décembre 2019 afin de recenser toute nouvelle étude pertinente. Les recherches ont été limitées à la langue anglaise et à l'année de publication de 1980 à aujourd'hui, sauf pour la base de données Scopus, qui a été consultée à partir de 1979, l'année 1980 n'étant pas sélectionnable. Les listes de références des études incluses ont été scannées pour s'assurer de l'intégralité de la recherche et exportées dans Endnote x8.2. 


\section{Critères d'admissibilité}

Les études incluses sont celles qui ont été publiées en anglais et concernaient les soins infirmiers de recherche clinique en oncologie sur au moins un de ces thèmes : rôle, lignes directrices/cadre de pratique, compétences, pratique avancée, milieu de pratique. Les éditoriaux, commentaires, résumés de conférences et études indiquant en milieux non oncologiques ont été exclus.

\section{Sélection d'études}

Comme indiqué dans le graphique PRISMA (figure 1), 1307 documents sont ressortis de la première recherche. Après la suppression des doublons, 771 titres et résumés ont été survolés, puis 211 articles ont été sélectionnés pour une revue du texte intégral. Au total, 182 études ont ensuite été exclues pour diverses raisons (voir figure 1), et 29 ont donc été incluses dans la présente revue.

\section{Extraction et consignation des résultats en graphique}

Lextraction et la consignation des résultats ont été effectuées par MH. Les données suivantes ont été extraites de chaque article : auteur, titre, année de publication, pays d'origine, buts et objectifs de l'étude, population étudiée, taille des échantillons, caractéristiques

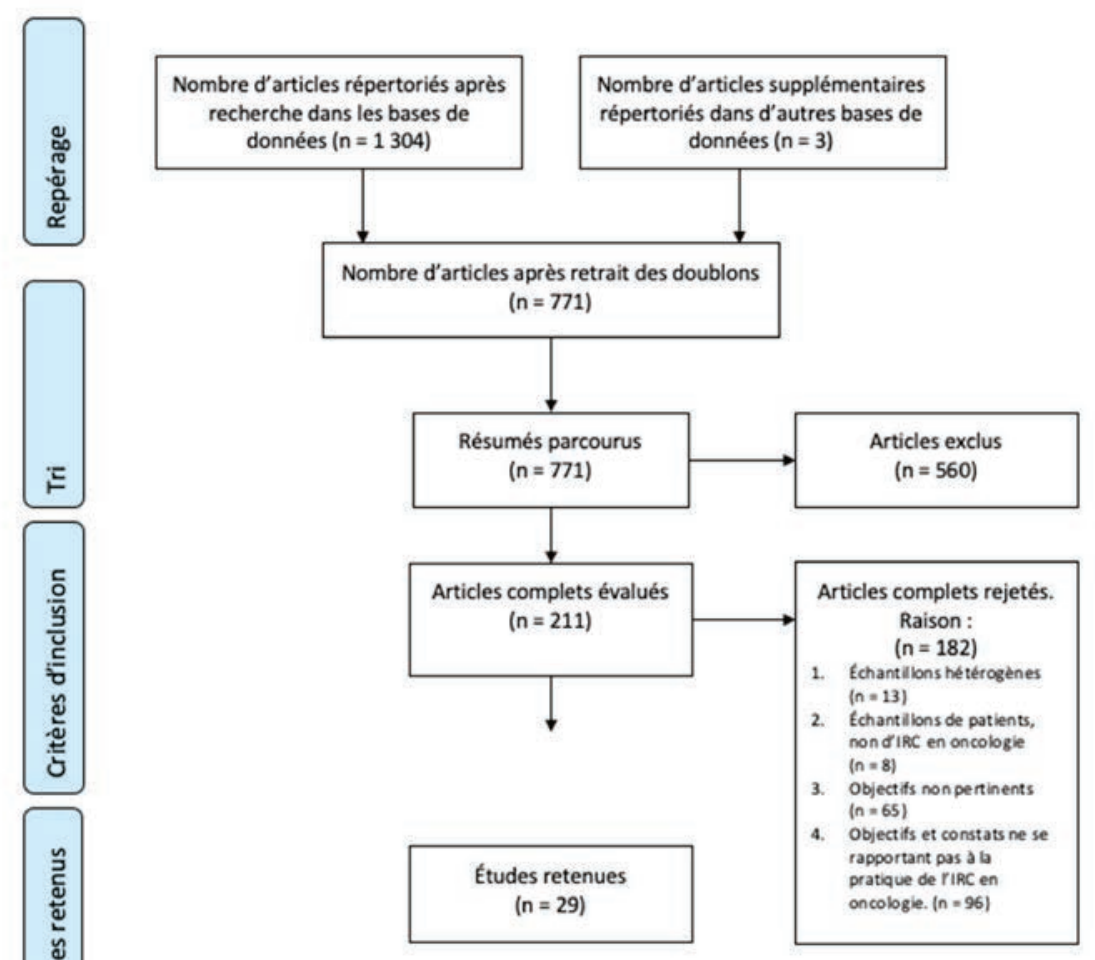
des participants (infirmière ou non, milieu oncologique ou non), intervention, contexte de pratique (patient hospitalisé ou non). La mise en tableau des principaux constats émanant des données extraites a formé un résumé descriptif des résultats. Guidés par la question de la recherche et les critères d'admissibilité, les thèmes reflétant la pratique des IRC en oncologie ont été déterminés et catégorisés par l'auteure principale en étroite consultation avec les autres membres de l'équipe.

\section{RÉSULTATS}

$\mathrm{Au}$ total, 29 études répondaient aux critères d'inclusion (tableau 1). Les thèmes recensés ont été regroupés selon les thématiques suivantes : description du rôle ( $n=11$ études), compétences ( $n=3$ études), éducation et formation ( $n=5$ études), charge de travail ( $n=3$ études), pratique avancée ( $n=4$ études), et perspectives internationales ( $n=3$ études) (tableau 1).

\section{Perspectives internationales \\ Description du rôle}

Les thèmes portant sur la description du rôle ont semblé prédominants et ont été organisés en sous-thèmes : description initiale du rôle, élaboration d'un outil de délimitation de rôle, contexte de pratique en oncologie pédiatrique, définition du domaine de pratique, et identité professionnelle de l'IRC en oncologie.

\section{Description initiale du rôle de l'IRC en oncologie}

La documentation du rôle de l'IRC en oncologie remonte au début des années 1980. Le rôle et les responsabilités de ce type d'infirmière de recherche consistaient alors à éduquer le patient, défendre ses droits, obtenir un consentement éclairé et participer à la collecte ainsi qu'à l'analyse de données (Hubbard et Donehower, 1980). Au début, la description du rôle de l'IRC en oncologie était générale avec des responsabilités comme la gestion des patients de la recherche, la mise en œuvre des essais cliniques et la participation à la mise en œuvre (Hubbard et Donehower, 1980).

Les clarifications du rôle et des responsabilités de l'IRC sont devenues plus évidentes dans les années 2000 avec le travail d'Ocker et Plank (2000), lorsqu'une initiative a été entreprise pour définir le rôle de manière empirique et distinguer son orientation par rapport aux autres rôles infirmiers. La nécessité de définir et de délimiter le rôle de l'IRC en oncologie était en partie le résultat d'une demande croissante de main-d'œuvre, de besoins accrus de l'IRC en oncologie et d'un ensemble croissant de preuves de la pratique. Les soins directs aux patients liés aux exigences du protocole ont été soulignés comme une partie essentielle du rôle de l'IRC en oncologie. L'examen du rôle de l'IRC en oncologie, de l'infirmière clinicienne spécialisée et de l'infirmière en pratique avancée a montré que chacun d'entre eux était un rôle de la pratique avancée (Ocker et Plank, 2000). 


\begin{tabular}{|c|c|c|c|c|c|}
\hline \multicolumn{6}{|c|}{ Tableau 1. Caractéristiques de l'étude } \\
\hline $\begin{array}{l}\text { Auteur } \\
\text { principal / } \\
\text { année / pays }\end{array}$ & Devis et objectifs de l'étude & $\begin{array}{l}\text { Échantillon de } \\
\text { l'étude }\end{array}$ & Intervention & Principaux constats & Thème \\
\hline $\begin{array}{l}\text { Hubbard, } \\
1980 \\
\text { É.-U. }\end{array}$ & $\begin{array}{l}\text { Empirique. } \\
\text { Réflexions sur le rôle de l'infirmière de } \\
\text { recherche clinique et des propositions } \\
\text { de description de rôle qui favoriseront } \\
\text { une plus grande intégration des } \\
\text { infirmières dans la recherche clinique. }\end{array}$ & $\begin{array}{l}\text { IRC en } \\
\text { oncologie }\end{array}$ & $\begin{array}{l}\text { Différenciation des } \\
\text { rôles des infirmières } \\
\text { cliniques, par rapport } \\
\text { aux infirmières des } \\
\text { soins intégraux } \\
\text { et des infirmières } \\
\text { spécialisées. }\end{array}$ & $\begin{array}{l}\text { Discussion sur le contexte pratique du } \\
\text { cadre des essais cliniques en oncologie } \\
\text { et des caractéristiques des essais de } \\
\text { phase I, II et III dans lesquels les rôles } \\
\text { des IRC sont guidés pour répondre } \\
\text { aux besoins des patients. Propositions } \\
\text { sur la description et l'expansion des } \\
\text { rôles. }\end{array}$ & $\begin{array}{l}\text { Description du } \\
\text { rôle }\end{array}$ \\
\hline $\begin{array}{l}\text { Ocker, } 2000 \\
\text { Royaume-Uni }\end{array}$ & $\begin{array}{l}\text { Étude de cas descriptive. } \\
\text { Description du cadre pour l'élaboration } \\
\text { du rôle de l'infirmière de recherche au } \\
\text { sein du programme de recherche en } \\
\text { oncologie dans une clinique externe } \\
\text { d'oncologie. }\end{array}$ & $\begin{array}{l}\text { IRC en } \\
\text { oncologie }\end{array}$ & $\begin{array}{l}\text { Restructuration } \\
\text { du programme } \\
\text { de recherche } \\
\text { et de formation } \\
\text { du personnel en } \\
\text { oncologie. }\end{array}$ & $\begin{array}{l}\text { Examen du rôle de l'IRC et des limites } \\
\text { de son rôle avec le rôle de l'infirmière } \\
\text { clinique spécialisée et de l'infirmière } \\
\text { en pratique avancée. }\end{array}$ & $\begin{array}{l}\text { Description du } \\
\text { rôle }\end{array}$ \\
\hline $\begin{array}{l}\text { Ehrenberger, } \\
2004 \\
\text { É.U. }\end{array}$ & $\begin{array}{l}\text { Transversale descriptive. } \\
\text { Un groupe de travail a rédigé le } \\
\text { questionnaire de l'IRC ("CTNQ ») } \\
\text { comme outil pour mesurer les activités } \\
\text { infirmières dans les essais cliniques, } \\
\text { leur fréquence et leur importance. }\end{array}$ & $\begin{array}{l}\text { IRC en } \\
\text { oncologie } \\
n=40\end{array}$ & Non disponible & $\begin{array}{l}\text { Élaboration du questionnaire CTNQ } \\
\text { de l'infirmière sur les essais cliniques. }\end{array}$ & $\begin{array}{l}\text { Description du } \\
\text { rôle }\end{array}$ \\
\hline $\begin{array}{l}\text { Coulson, } \\
2000 \\
\text { Royaume-Uni }\end{array}$ & $\begin{array}{l}\text { Empirique. } \\
\text { Description de différentes facettes } \\
\text { du rôle de l'IRC dans la recherche } \\
\text { clinique en oncologie pédiatrique au } \\
\text { Royaume-Uni. }\end{array}$ & $\begin{array}{l}\text { IRC en } \\
\text { oncologie } \\
\text { pédiatrique }\end{array}$ & Non rapportée & $\begin{array}{l}\text { Relevé des caractéristiques uniques } \\
\text { dans les essais cliniques pédiatriques } \\
\text { par rapport aux essais chez les adultes, } \\
\text { et description du rôle de l'IRC en } \\
\text { milieu pédiatrique. }\end{array}$ & $\begin{array}{l}\text { Description du } \\
\text { rôle }\end{array}$ \\
\hline $\begin{array}{l}\text { Catania, } \\
2008 \\
\text { Italie } \\
\end{array}$ & $\begin{array}{l}\text { Psychométrique. } \\
\text { Traduction en italien puis évaluation du } \\
\text { questionnaire CTNQ. }\end{array}$ & $\begin{array}{l}\text { IRC en } \\
\text { oncologie } \\
n=30\end{array}$ & Non rapportée & $\begin{array}{l}\text { Le test a confirmé la fiabilité du } \\
\text { questionnaire après sa traduction en } \\
\text { italien. }\end{array}$ & $\begin{array}{l}\text { Description du } \\
\text { rôle }\end{array}$ \\
\hline $\begin{array}{l}\text { Chang, } 2008 \\
\text { Canada }\end{array}$ & $\begin{array}{l}\text { Méthodologie mixte. } \\
\text { Exploration de la perception de } \\
\text { l'infirmière en pédiatrie sur : 1) les buts, } \\
\text { les objectifs, le rôle de l'infirmière, le } \\
\text { consentement éclairé, le processus } \\
\text { décisionnel de la phase I des essais } \\
\text { cliniques en oncologie pédiatrique; } \\
\text { 2) les facteurs qui influencent la } \\
\text { perception de l'infirmière en oncologie } \\
\text { pendant la phase I des essais cliniques. }\end{array}$ & $\begin{array}{l}\text { IRC en } \\
\text { oncologie } \\
n=43 \\
\text { travaillant } \\
\text { dans une unité } \\
\text { oncologique } \\
\text { dans un hôpital } \\
\text { pédiatrique } \\
\text { conjuguant } \\
\text { un espace } \\
\text { pour patients } \\
\text { hospitalisés et } \\
\text { une clinique } \\
\text { externe. }\end{array}$ & Non rapportée & $\begin{array}{l}\text { L'étude a porté sur les infirmières } \\
\text { travaillant dans les essais cliniques de } \\
\text { phase I (pédiatrie); elle ne mentionne } \\
\text { pas les infirmières de recherche } \\
\text { clinique, ce qui laisse supposer que } \\
\text { ce rôle n'a pas été développé dans } \\
\text { cet établissement, et que les soins } \\
\text { des études pédiatriques de phase I } \\
\text { en oncologie sont partagés entre les } \\
\text { infirmières de chevet et les autres } \\
\text { (infirmière clinicienne spécialisée, } \\
\text { infirmière clinicienne, infirmière } \\
\text { en soins intégraux dans un service } \\
\text { de consultation externe, infirmière } \\
\text { pédagogue, infirmière gestionnaire). }\end{array}$ & $\begin{array}{l}\text { Description du } \\
\text { rôle }\end{array}$ \\
\hline
\end{tabular}




\begin{tabular}{|c|c|c|c|c|c|}
\hline $\begin{array}{l}\text { Nagel, } 2010 \\
\text { É.-U./ } \\
\text { Canada }\end{array}$ & $\begin{array}{l}\text { Quantitatif empirique. } \\
\text { Caractérisation du rôle de l'IRC dans } \\
\text { un groupe coopératif d'essais cliniques. }\end{array}$ & $\begin{array}{l}n=85 \\
\text { (Répondants } \\
\text { exerçant à } \\
\text { la fois les } \\
\text { fonctions } \\
\text { d'infirmière et } \\
\text { d'IRC.) }\end{array}$ & Non rapportée & $\begin{array}{l}\text { Caractérisation des infirmières } \\
\text { dans le double rôle d'IRC et de } \\
\text { coordonnateur de recherche clinique; } \\
\text { valeur de la formation continue dans le } \\
\text { rendement professionnel des IRC. }\end{array}$ & $\begin{array}{l}\text { Description du } \\
\text { rôle }\end{array}$ \\
\hline $\begin{array}{l}\text { Catania, } \\
2012 \\
\text { Italie }\end{array}$ & $\begin{array}{l}\text { Empirique, qualitatif. } \\
\text { Délimitation du rôle de l'IRC en Italie, } \\
\text { en mettant l'accent sur deux aspects } \\
\text { : 1) évaluation du rôle; } 2 \text { ) qualité du } \\
\text { travail effectué. }\end{array}$ & $\begin{array}{l}\text { IRC en } \\
\text { oncologie } \\
n=30 \\
\text { travaillant dans } \\
\text { des hôpitaux } \\
\text { spécialisés en } \\
\text { cancérologie }\end{array}$ & Non rapportée & $\begin{array}{l}\text { Les IRC en Italie sont pleinement } \\
\text { conscientes d'être des membres clés } \\
\text { des équipes des équipes de recherche, } \\
\text { potentiellement capables de partager } \\
\text { les objectifs des études cliniques, } \\
\text { ainsi que de soutenir l'intégrité des } \\
\text { protocoles et la sécurité des patients. } \\
\text { La participation des IRC à la gestion } \\
\text { des essais cliniques est faible. }\end{array}$ & $\begin{array}{l}\text { Description du } \\
\text { rôle }\end{array}$ \\
\hline $\begin{array}{l}\text { Choi, } 2018 \\
\text { Corée }\end{array}$ & $\begin{array}{l}\text { Psychométrique } \\
\text { Validation d'un questionnaire pour } \\
\text { délimiter les rôles infirmiers dans les } \\
\text { essais cliniques en Corée. }\end{array}$ & $\begin{array}{l}\text { n = } 53 \text { IRC } \\
\text { travaillant dans } \\
\text { des centres de } \\
\text { cancérologie } \\
\text { clinique de } \\
\text { cinq hôpitaux } \\
\text { universitaires. }\end{array}$ & Non rapportée & $\begin{array}{l}\text { La version coréenne du questionnaire } \\
\text { CTNQ de l'infirmière de recherche } \\
\text { clinique est testée et jugée fiable, } \\
\text { valide et interchangeable avec le } \\
\text { questionnaire original. }\end{array}$ & $\begin{array}{l}\text { Description du } \\
\text { rôle }\end{array}$ \\
\hline $\begin{array}{l}\text { Lubejko, } 2011 \\
\text { É.-U. }\end{array}$ & $\begin{array}{l}\text { Avis d'experts } \\
\text { Établissement des compétences de } \\
\text { base requises d'une infirmière novice } \\
\text { en oncologie pour les essais cliniques } \\
\text { dans divers contextes. }\end{array}$ & $\begin{array}{l}\text { IRC en } \\
\text { oncologie }\end{array}$ & Non rapportée & $\begin{array}{l}\text { Lignes directrices sur les compétences } \\
\text { pour l'IRC novice en oncologie. }\end{array}$ & Compétences \\
\hline $\begin{array}{l}\text { Scott, } 2012 \\
\text { Australie }\end{array}$ & $\begin{array}{l}\text { Statistique, descriptif. } \\
\text { Élaboration et mise à l'essai } \\
\text { d'un questionnaire mesurant les } \\
\text { connaissances et les compétences } \\
\text { des infirmières chargées des essais } \\
\text { cliniques sur le cancer en Australie; } \\
\text { et évaluation des connaissances et } \\
\text { compétences de l'IRC en Australie } \\
\text { pour mieux comprendre leurs } \\
\text { besoins en matière d'éducation et de } \\
\text { formation. }\end{array}$ & $\begin{array}{l}\text { IRC en } \\
\text { oncologie } \\
n=61\end{array}$ & Non rapportée & $\begin{array}{l}\text { 1) Les connaissances déclarées } \\
\text { sont faibles dans les domaines liés } \\
\text { aux méthodes et à la conduite } \\
\text { des essais cliniques; } 2 \text { ) Les } \\
\text { connaissances déclarées n'étaient } \\
\text { pas significativement liées aux } \\
\text { qualifications d'infirmières, aux } \\
\text { qualifications post-universitaires ou } \\
\text { à l'importance perçue; 3) Les années } \\
\text { d'expérience en tant qu'IRC étaient } \\
\text { significativement et positivement liées } \\
\text { aux connaissances déclarées. }\end{array}$ & Compétences \\
\hline $\begin{array}{l}\text { Arrigo, } 1994 \\
\text { Europe } \\
\text { (Royaume- } \\
\text { Uni, } \\
\text { Pays-Bas, } \\
\text { Belgique, } \\
\text { France, } \\
\text { Espagne, } \\
\text { Grèce) }\end{array}$ & $\begin{array}{l}\text { Statistique, descriptif. } \\
\text { Recensement et identification des } \\
\text { infirmières participant aux essais } \\
\text { cliniques en oncologie, pour décrire } \\
\text { l'étendue de leur participation et } \\
\text { documenter leurs besoins spécifiques. }\end{array}$ & $\begin{array}{l}n=120 \\
\text { infirmières } \\
\text { autorisées de six } \\
\text { pays européens }\end{array}$ & Non rapportée & $\begin{array}{l}\text { Les IRC classent l'éducation et la } \\
\text { formation parmi les ressources les } \\
\text { plus importantes pour leur travail. } \\
\text { Les infirmières se sont senties } \\
\text { modérément satisfaites de leurs } \\
\text { connaissances. La participation des } \\
\text { infirmières aux essais cliniques dans les } \\
\text { pays européens est faible. }\end{array}$ & Compétences \\
\hline
\end{tabular}




\begin{tabular}{|c|c|c|c|c|c|}
\hline $\begin{array}{l}\text { Liptrott, } \\
2009 \\
\text { Italie }\end{array}$ & $\begin{array}{l}\text { Étude de cas. } \\
\text { Offre d'un programme d'enseignement } \\
\text { et de soutien pour intégrer deux } \\
\text { infirmières dans le rôle de l'infirmière } \\
\text { de recherche. }\end{array}$ & $\begin{array}{l}n=2 \\
\text { Infirmières } \\
\text { de service } \\
\text { d'hospitalisation } \\
\text { qui deviennent } \\
\text { des infirmières } \\
\text { de recherche }\end{array}$ & Non rapportée & $\begin{array}{l}\text { Les infirmières de recherche suivant } \\
\text { le programme ont toujours souhaité } \\
\text { avoir plus de connaissances à la fin. La } \\
\text { recommandation sur les compétences } \\
\text { de l'IRC émanant de la littérature ne } \\
\text { se reflétait pas nécessairement dans } \\
\text { les besoins de l'établissement local. }\end{array}$ & Compétences \\
\hline $\begin{array}{l}\text { Scott, } 2013 \\
\text { Australie }\end{array}$ & $\begin{array}{l}\text { Questionnaire } \\
\text { Visée de mieux comprendre les besoins } \\
\text { en matière d'éducation et de formation } \\
\text { des infirmières chargées des essais } \\
\text { cliniques en Australie. }\end{array}$ & $\begin{array}{l}\text { IRC en } \\
\text { oncologie } n=61\end{array}$ & Non rapportée & $\begin{array}{l}\text { La majorité des participants sont soit } \\
\text { titulaires d'un diplôme de troisième } \\
\text { cycle, soit très intéressés à suivre } \\
\text { cette formation. Il est recommandé } \\
\text { d'obtenir un diplôme de troisième } \\
\text { cycle pour répondre aux besoins } \\
\text { d'éducation et de formation des IRC. }\end{array}$ & $\begin{array}{l}\text { Éducation/ } \\
\text { formation }\end{array}$ \\
\hline $\begin{array}{l}\text { Showalter, } \\
2017 \\
\text { E.-U. }\end{array}$ & $\begin{array}{l}\text { Étude de cas. } \\
\text { Afin d'atténuer certains des problèmes } \\
\text { liés au recrutement et à la fidélisation } \\
\text { de la main-d'œuvre, un programme de } \\
\text { résidence des infirmières de recherche } \\
\text { a été élaboré et mis en œuvre en } 2016 .\end{array}$ & $\begin{array}{l}\text { IRC en } \\
\text { oncologie }\end{array}$ & $\begin{array}{l}\text { Le programme } \\
\text { comprend une } \\
\text { orientation de } 4 \\
\text { mois suivie d'un ou } \\
\text { deux jours de classe } \\
\text { de résidence sur une } \\
\text { période de } 12 \text { mois. }\end{array}$ & $\begin{array}{l}\text { Le programme d'études comprenait } \\
\text { une formation en oncologie et } \\
\text { en essais cliniques, soulignant à } \\
\text { nouveau l'intégration impérative de } \\
\text { la formation en oncologie dans la } \\
\text { préparation des IRC. Les premières } \\
\text { réactions des participants et des } \\
\text { services d'embauche ont été positives, } \\
\text { avec une grande volonté d'attirer de } \\
\text { nouveaux résidents. }\end{array}$ & $\begin{array}{l}\text { Éducation/ } \\
\text { formation }\end{array}$ \\
\hline $\begin{array}{l}\text { Herena, } \\
2018 \\
\text { E.-U. }\end{array}$ & $\begin{array}{l}\text { Étude de cas. } \\
\text { Rapport sur le développement et la } \\
\text { mise en place d'un cours de formation } \\
\text { pour les IRC nouvellement recrutées, } \\
\text { basé sur la norme American Nurse } \\
\text { Association's Scope and Standards of } \\
\text { Practice pour les soins infirmiers en } \\
\text { recherche clinique. }\end{array}$ & $\begin{array}{l}\text { IRC en } \\
\text { oncologie }\end{array}$ & $\begin{array}{l}\text { Cours de formation } \\
\text { destiné aux infirmières } \\
\text { de recherche clinique } \\
\text { nouvellement } \\
\text { recrutées et en place. }\end{array}$ & $\begin{array}{l}\text { Avec la mise en place du cours, il } \\
\text { y a eu une diminution du taux de } \\
\text { rotation et une augmentation du taux } \\
\text { de rétention au cours du premier } \\
\text { trimestre de l'exercice financier, après } \\
\text { l'intervention. }\end{array}$ & $\begin{array}{l}\text { Éducation/ } \\
\text { formation }\end{array}$ \\
\hline $\begin{array}{l}\text { Bird, } 2005 \\
\text { Royaume-Uni }\end{array}$ & $\begin{array}{l}\text { Revue de la littérature } \\
\text { Examen du rôle de l'IRC } \\
\text { comparativement à la pratique avancée } \\
\text { des soins infirmiers. }\end{array}$ & Non rapporté & Non rapportée & $\begin{array}{l}\text { Établissement d'un cadre et du rôle } \\
\text { potentiel pour la pratique avancée. }\end{array}$ & $\begin{array}{l}\text { Pratique } \\
\text { avancée }\end{array}$ \\
\hline $\begin{array}{l}\text { Rosenzweig, } \\
2005 \\
\text { É.-U. }\end{array}$ & $\begin{array}{l}\text { Descriptif. } \\
\text { Discussion du processus, des } \\
\text { considérations, des avantages et des } \\
\text { défis de l'infirmière agissant comme } \\
\text { chercheur principal dans un essai } \\
\text { pharmacologique contre le cancer. }\end{array}$ & $\begin{array}{l}\text { Infirmière } \\
\text { co-chercheuse }\end{array}$ & Non rapportée & $\begin{array}{l}\text { Exemple de la manière dont les } \\
\text { infirmières participant à des essais } \\
\text { cliniques peuvent faire progresser leur } \\
\text { pratique et être impliquées dans des } \\
\text { essais cliniques en tant que chercheur } \\
\text { principal. }\end{array}$ & $\begin{array}{l}\text { Pratique } \\
\text { avancée }\end{array}$ \\
\hline $\begin{array}{l}\text { Winter, } 2012 \\
\text { É.-U. }\end{array}$ & $\begin{array}{l}\text { Quantitatif. } \\
\text { Étude d'une clinique nouvellement } \\
\text { créée, dirigée par des infirmières, } \\
\text { pour les essais cliniques sur le cancer } \\
\text { gastro-intestinal. }\end{array}$ & $\begin{array}{l}n=35 \\
\text { Patients avec } \\
\text { un cancer } \\
\text { gastro-intestinal }\end{array}$ & $\begin{array}{l}\text { Clinique dirigée } \\
\text { par des infirmières } \\
\text { praticiennes }\end{array}$ & $\begin{array}{l}\text { Grande satisfaction des patients des } \\
\text { essais cliniques grâce à la clinique } \\
\text { dirigée par des infirmières. }\end{array}$ & $\begin{array}{l}\text { Pratique } \\
\text { avancée }\end{array}$ \\
\hline
\end{tabular}




\begin{tabular}{|c|c|c|c|c|c|}
\hline $\begin{array}{l}\text { Milani, } 2017 \\
\text { Italie }\end{array}$ & $\begin{array}{l}\text { Mesure de la charge de travail. } \\
\text { Description d'un outil permettant } \\
\text { de mesurer la charge de travail de } \\
\text { l'IRC exprimée en temps consacré } \\
\text { à l'accomplissement des activités de } \\
\text { base. }\end{array}$ & $\begin{array}{l}\text { IRC en } \\
\text { oncologie } n=7\end{array}$ & Non rapportée & $\begin{array}{l}\text { Mesure de la charge de travail et } \\
\text { développement de l'outil d'acuité } \\
\text { protocolaire du programme Wichita } \\
\text { Community Clinical Oncology } \\
\text { (WCCOP). }\end{array}$ & $\begin{array}{l}\text { Charge de } \\
\text { travail }\end{array}$ \\
\hline $\begin{array}{l}\text { Lee, } 2018 \\
\text { Corée }\end{array}$ & $\begin{array}{l}\text { Quantitatif. } \\
\text { Mesure de la valeur relative du travail } \\
\text { des IRC par le calcul de leur charge de } \\
\text { travail.. }\end{array}$ & $n=70$ IRC & $\begin{array}{l}\text { Comparaison de la } \\
\text { différence de valeur } \\
\text { relative du travail } \\
\text { entre l'oncologie, } \\
\text { la cardiologie et } \\
\text { l'endocrinologie. }\end{array}$ & $\begin{array}{l}\text { Développement d'un outil de mesure } \\
\text { de la charge de travail. Par rapport } \\
\text { aux essais en cardiologie et en } \\
\text { endocrinologie, les essais cliniques } \\
\text { en oncologie sont plus intensifs et } \\
\text { nécessitent plus de temps et de travail. }\end{array}$ & $\begin{array}{l}\text { Charge de } \\
\text { travail }\end{array}$ \\
\hline $\begin{array}{l}\text { Good, } 2013 \\
\text { É.-U. }\end{array}$ & $\begin{array}{l}\text { Étude de cas. } \\
\text { Décrire l'outil d'évaluation de la charge } \\
\text { de travail basé sur l'acuité qui facilite } \\
\text { l'évaluation et l'équilibre de la charge } \\
\text { de travail au sein du personnel infirmier } \\
\text { de recherche. }\end{array}$ & $\begin{array}{l}\text { IRC en } \\
\text { oncologie }\end{array}$ & Non rapportée & $\begin{array}{l}\text { Compte rendu de l'outil d'évaluation } \\
\text { de la charge de travail basé sur l'acuité } \\
\text { qui facilite l'évaluation et l'équilibre de } \\
\text { la charge de travail parmi le personnel } \\
\text { infirmier de recherche. }\end{array}$ & $\begin{array}{l}\text { Charge de } \\
\text { travail }\end{array}$ \\
\hline $\begin{array}{l}\text { Fujiwara, } \\
2017 \\
\text { Japon }\end{array}$ & $\begin{array}{l}\text { Qualitatif. } \\
\text { Clarification de la coordination et de la } \\
\text { pratique des soins aux patients fournis } \\
\text { par les coordonnateurs de recherche } \\
\text { clinique dans les essais cliniques de } \\
\text { phase I sur le cancer au Japon, et } \\
\text { compréhension du point de vue des } \\
\text { coordonnateurs de recherche clinique } \\
\text { sur les attentes des patients et leur } \\
\text { compréhension de ces essais. }\end{array}$ & $\begin{array}{l}n=15(13 \\
\text { infirmières et } \\
2 \text { pharmaciens })\end{array}$ & Non rapportée & $\begin{array}{l}\text { Les besoins des patients dans les } \\
\text { essais oncologiques de phase I } \\
\text { ont été examinés sous l'angle des } \\
\text { coordonnateurs de recherche clinique. } \\
\text { Les différents besoins des patients } \\
\text { ont été déterminés dans les trois } \\
\text { phases d'une étude : la phase de } \\
\text { recrutement et de consentement, } \\
\text { pendant l'intervention de l'essai, et } \\
\text { pendant les interventions post-essai. } \\
\text { Les patients dans cette phase ont } \\
\text { besoin d'un soutien psychosocial } \\
\text { important, un domaine de formation } \\
\text { qui serait utile donc pour les } \\
\text { coordonnateurs de recherche clinique. } \\
\text { Les coordonnateurs de recherche } \\
\text { clinique ont un bagage professionnel } \\
\text { varié : soins infirmiers, laboratoire, } \\
\text { pharmacologie, etc. }\end{array}$ & $\begin{array}{l}\text { Perspectives } \\
\text { internationales }\end{array}$ \\
\hline $\begin{array}{l}\text { Matsumoto, } \\
2011 \\
\text { Japon }\end{array}$ & $\begin{array}{l}\text { Qualitatif. } \\
\text { Exploration des difficultés rencontrées } \\
\text { par le personnel infirmier dans son } \\
\text { travail lors des essais oncologiques de } \\
\text { phase I. }\end{array}$ & $\begin{array}{l}n=21 \\
\text { échantillon de } \\
\text { commodité } \\
\text { d'infirmières; } \\
\text { aucune IRC } \\
\text { et aucun } \\
\text { participant } \\
\text { n'avait travaillé } \\
\text { en tant que } \\
\text { coordonnateur } \\
\text { de recherche } \\
\text { clinique ou } \\
\text { était infirmière } \\
\text { spécialisée } \\
\text { certifiée }\end{array}$ & Non rapportée & $\begin{array}{l}\text { 1) Le personnel infirmier général } \\
\text { devait fournir des soins infirmiers } \\
\text { aux patients participant aux essais } \\
\text { oncologiques de phase I en plus des } \\
\text { soins aux patients ne participant pas } \\
\text { aux essais et avait des difficultés à } \\
\text { assumer ces deux rôles. 2) Il y avait } \\
\text { une déconnexion entre les infirmières } \\
\text { et le reste de l'équipe d'essai clinique } \\
\text { alors que les infirmières ont le plus de } \\
\text { contacts avec les sujets de recherche. } \\
\text { Peu de place laissée aux infirmières } \\
\text { pour qu'elles s'expriment et } \\
\text { s'impliquent dans le fonctionnement } \\
\text { des essais cliniques, la prise de } \\
\text { décision, ainsi que l'expertise et la } \\
\text { connaissance des essais cliniques. }\end{array}$ & $\begin{array}{l}\text { Perspectives } \\
\text { internationales }\end{array}$ \\
\hline $\begin{array}{l}\text { Cheng, } 1998 \\
\text { Taiwan }\end{array}$ & $\begin{array}{l}\text { Avis d'experts } \\
\text { Rapport sur la mise en œuvre du rôle } \\
\text { d'IRC dans les essais cliniques en } \\
\text { oncologie à Taiwan. }\end{array}$ & $\begin{array}{l}\text { IRC en } \\
\text { oncologie }\end{array}$ & Non rapportée & $\begin{array}{l}\text { La mise en œuvre du rôle de l'IRC à } \\
\text { Taiwan n'a pas bien réussi en 1998, } \\
\text { laissant la pratique sans soutien et } \\
\text { créant, par conséquent, un taux de } \\
\text { rotation élevé des IRC. }\end{array}$ & $\begin{array}{l}\text { Perspectives } \\
\text { internationales }\end{array}$ \\
\hline
\end{tabular}


Questionnaire «Clinical Trial Nurse » comme outil de délimitation des rôles

Le Clinical Trial Nurse Questionnaire (questionnaire de l'infirmière de recherche clinique en oncologie [CTNQ]) a été le premier outil conçu pour délimiter le rôle de l'IRC en oncologie (Ehrenberger et Lillington, 2004). Au début des années 2000, des IRC de l'Oncology Nursing Society des États-Unis ont formé un groupe d'intérêt spécial. qui a mené un sondage ayant mené à l'élaboration du CTNQ en oncologie (Ehrenberger et Lillington, 2004). Le questionnaire contenait douze sections définissant les activités, les responsabilités et les divers aspects de la pratique des IRC en oncologie. Il a été structuré comme un instrument d'auto-évaluation décrivant les rôles des infirmières en fonction de la performance, la fréquence et la perception de l'importance. Sur les douze sections, huit portaient sur le rôle de l'infirmière : évaluation $\mathrm{du}$ protocole, planification du protocole, recrutement des participants, processus de consentement éclairé, produit de recherche, mise en œuvre et évaluation, gestion des données, et application du rôle de l'infirmière professionnelle. Dans les quatre autres sections, on cherche à évaluer les aspects du rôle et de la pratique de l'IRC en oncologie. Sont couvertes : la perception et l'expérience de l'infirmière, les caractéristiques démographiques, les caractéristiques professionnelles, et les caractéristiques de l'organisation du travail (Ehrenberger et Lillington, 2004).

Le CTNQ a aidé à préciser la définition du rôle et la pratique de l'IRC en oncologie tout en abordant certaines questions liées à l'identité professionnelle et à l'ambiguïté du rôle de l'époque. L'outil a été bien reçu par la profession, tant à l'échelle nationale qu'internationale. Il a été traduit en italien et en coréen, puis est devenu une norme pour d'autres études sur la définition du rôle de l'IRC (Catania et al., 2012; Catania, Poire, Dozin, Bernardi et Boni, 2008; Choi et Park, 2018; Nagel, Gender et Bonner, 2010). Grâce à la réponse internationale positive et à l'adoption de cet outil, la pratique émergente de l'IRC en oncologie a été de plus en plus reconnue mondialement en tant que rôle infirmer spécialisé.

Pratique de l'IRC en oncologie dans les essais cliniques en oncologie pédiatrique de phase I et II

Les soins infirmiers de recherche clinique en milieu pédiatrique ont fait l'objet d'une littérature distincte. Le milieu pédiatrique constitue un contexte de pratique unique en ce qui concerne le consentement éclairé, le recrutement de sujets parmi les populations vulnérables et la participation importante des parents et des familles à la prise de décision (Coulson et Phelan, 2000). Les essais cliniques de phase I exigent généralement beaucoup de personnel, en particulier avec des sujets pédiatriques, lorsqu'une première expérimentation sur l'humain d'un nouveau traitement est réalisée sur des enfants. Pour cette jeune population, l'incidence et les manifestations des effets indésirables sont diverses en raison de la taille relativement petite de l'échantillon de l'étude, du stade de développement des sujets et de leur tolérance et de leur capacité, ce qui justifie souvent des évaluations de sécurité et des observations supplémentaires (Carlson, Reilly et Hitchens, 2005; Coulson et Phelan, 2000). Pour ces raisons, les essais cliniques en pédiatrie ont généralement eu lieu dans des établissements permettant l'hospitalisation, par opposition aux cliniques externes et aux centres de recherche universitaires (Carlson et al., 2005; Nagel et al., 2010).

Au départ, le rôle de l'IRC en oncologie pédiatrique a été considéré comme un prolongement du rôle de l'infirmière en oncologie pédiatrique. Au début, alors que peu de nouveaux essais cliniques pédiatriques étaient disponibles, un effort pour maximiser l'allocation des ressources tout en répondant aux besoins constants de la recherche a placé les infirmières en oncologie pédiatrique dans une position idéale pour participer aux soins des patients pédiatriques inscrits dans les essais cliniques. Les titres de poste des infirmières assumant le rôle d'IRC en oncologie étaient tout aussi ambigus ou incohérents au moment de la mise en place du rôle initial que chez les infirmières en milieu adulte. L'identité professionnelle et la confusion concernant le rôle ont été perçus comme des problèmes courants dans un environnement pédiatrique, comme c'était le cas dans d'autres domaines des soins infirmiers en recherche clinique (Carlson et al., 2005; Chang, 2008; Nagel et al., 2010).

Nagel, Gender et Bonner (2010) ont mené une étude sur le rôle d'IRC au sein du Children's Oncology Group (COG), en l'examinant de manière empirique à l'aide du CTNQ. Un échantillon de 85 répondants des États-Unis et du Canada, qui exerçaient des rôles combinés d'IRC et de coordonnateur de la recherche clinique (CRN), ont rempli le questionnaire et donné un aperçu du rôle de l'IRC en pédiatrie. Les résultats indiquent que le rôle de l'IRC en pédiatrie est spécialisé et nécessite des connaissances poussées, et un bon esprit critique et décisionnel pour répondre à la combinaison des exigences de l'IRC et du coordonnateur de la recherche clinique.

\section{Domaine et cadre de pratique}

Les études précédentes sur le rôle de l'IRC généraliste ont classé les infirmières travaillant dans les essais cliniques en deux groupes principaux - l'IRC axée sur la pratique clinique et la recherche, et l'infirmière coordonnatrice de recherche, axée sur la coordination de la recherche et la gestion des études (Bevans et al., 2011; Castro et al., 2012). Cependant, la confusion persistait, car l'ONS suggérait que le rôle de l'IRC en oncologie englobait la coordination des essais cliniques et la gestion des patients participant aux essais (ONS, 2010, 2016). L'ONS a reconnu que les cadres de pratique et les titres de postes d'IRC en oncologie étaient variés et pouvaient inclure, sans s'y limiter, les postes de coordonnatrice d'essais cliniques, d'infirmière de recherche clinique, d'infirmière coordonnatrice de recherche et de coordonnateur de protocoles. En tant que tel, l'IRC en oncologie peut ou non fournir des soins directs aux patients dans certains milieux de recherche (ONS, 2016).

Purdom, Petersen et Haas (2017) ont mené une étude transversale en ligne auprès d'infirmières en oncologie occupant le rôle de coordonnatrices de recherche, de fournisseurs de soins directs et d'infirmières à double rôle (fournissant des soins aux patients et coordonnant les essais) et ont signalé que l'IRC en oncologie assumait généralement un double rôle par rapport à l'IRC dans les groupes de patients atteints de maladies autres 
que le cancer. Les auteurs ont décrit la pratique oncologique comme multidimensionnelle : les infirmières consacrent plus de temps aux soins directs aux patients, aux processus de recherche, à la coordination des études, et les rôles et responsabilités sont plus complexes et diversifiés dans un milieu oncologique (Purdom et al., 2017). Purdom et collaborateurs (2017) ont élaboré un cadre décrivant le rôle de l'IRC en oncologie qui comprend les domaines suivants : soins, gestion d'études, expertise, direction, préparation, données, avancement de la science et éthique. Selon les affirmations et la vision de l'ONS sur l'oncologie, le rôle de l'IRC en oncologie et la pratique élaborés par Purdom et collaborateurs, les soins infirmiers de recherche clinique en oncologie sont reconnus comme une sous-spécialité distincte des soins infirmiers en oncologie en général et du rôle de l'infirmière de recherche clinique dans d'autres groupes de maladies.

\section{Complexité de l'identité professionnelle}

La confusion quant au rôle de l'IRC semble découler de l'incohérence et de la discordance des termes utilisés dans les titres et les descriptions de poste. Les descriptions d'emplois et de postes sont souvent rédigées en fonction des besoins spécifiques d'une agence privée et sont adaptées aux programmes de recherche locaux. L'utilisation de certains titres, comme gestionnaire de données ou coordonnateur de recherche clinique, n'était pas limitée à la profession d'infirmière, mais était accessible à d'autres professionnels (Purdom et al., 2017). La diversité et l'ambiguité des rôles étaient donc non seulement un obstacle quant à la précision des rôles, mais aussi un obstacle en matière d'identité professionnelle de l'IRC, d'image de marque et dans la communication du rôle aux patients et à la population. L'expression « infirmière de recherche clinique » est courante dans la littérature infirmière de la dernière décennie, en particulier dans les études importantes portant sur le rôle, les compétences, l'éducation ainsi que le domaine et le cadre de la pratique des IRC en oncologie, et apparait donc comme un titre dans les publications infirmières en oncologie (Ness et Royce, 2017; Purdom et al., 2017; Scott, White et Roydhouse, 2013).

\section{Compétences}

Le premier guide des compétences de base de l'IRC en oncologie a été élaboré en 2010 par l'ONS, en réponse à l'augmentation du nombre d'IRC en oncologie, aux besoins de formation sur les essais cliniques et à la normalisation du rôle (Lubejko et al., 2011). Ce guide a été mis à jour en 2016; on y a intégré de nouvelles données scientifiques sur le champ d'exercice de ce rôle spécialisé, et ajoutant des recommandations pour soutenir et clarifier les rôles et responsabilités. Dans neuf domaines fonctionnels, le guide énonce les attentes en matière de rôle et souligne les connaissances et compétences requises d'une IRC en oncologie (ONS, 2016), aux niveaux d'IRC en oncologie novice et expérimentée. La version de 2016 décrit aussi le rôle de leadership de l'IRC en oncologie et ajoute une définition de l'avancement de la pratique. Enfin, une mesure objective élaborée pour évaluer les compétences et l'expérience en pratique avancée, plutôt que le simple nombre d'années de travail en tant qu'IRC en oncologie, y est intégrée (ONS 2016).
Ce nouvel accent mis sur le leadership et l'avancement de la pratique concorde avec les données toujours plus nombreuses entourant la pratique avancée de l'IRC en oncologie. Bird et Kirshbaum (2005) ont proposé un cadre de la pratique avancée pour l'IRC en oncologie. Les auteurs ont suggéré que la longévité ou les années d'expérience seules ne devraient pas se traduire nécessairement par un niveau de compétence supérieur de la pratique, mais qu'il faudrait aussi que l'infirmière s'engage à se perfectionner pour faire progresser sa propre pratique. À ce titre, elle manifeste la capacité de traduire son expérience professionnelle en un niveau de réflexion avancé et utilise ces connaissances et compétences dans sa pratique quotidienne et dans son travail (Bird et Kirshbaum, 2005).

Par la suite, l'ONS a recommandé que la coordination des essais cliniques sur le cancer soit assurée le plus efficacement possible par une IRC en oncologie ayant une expérience des soins infirmiers en oncologie (ONS, 2016). Le guide de l'ONS 2016 met l'accent sur l'expérience des soins infirmiers en oncologie et les compétences en recherche clinique requises aux IRC en oncologie afin de répondre aux besoins uniques des patients cancéreux ainsi qu'au nombre élevé et à la complexité des essais cliniques sur le cancer. Au fil des ans, l'ONS a continué à répondre aux besoins de l'IRC en oncologie, permettant de mettre au point un outil et des exigences en matière de compétences qui définissent plus précisément la nature spécialisée de ce rôle.

\section{Éducation et formation}

Le cadre de la pratique, les compétences essentielles et autres documents de soutien similaires sur le rôle de l'IRC en oncologie sont des plans directeurs impératifs qui aident à structurer et à appuyer l'enseignement et la formation de l'IRC en oncologie lorsqu'ils sont intégrés dans les programmes détudes et d'orientation des infirmières nouvelles dans ce rôle. Les thèmes de l'éducation et de la formation ont été recensés dans cinq études publiées de 1994 à 2017 (tableau 1). L'objectif de ces programmes de formation varie, allant de l'amélioration de la qualité à la reconnaissance du rôle et de la contribution de l'IRC en oncologie, en passant par l'augmentation de l'appui à l'IRC en oncologie, le tout à la lumière du volume accru d'essais cliniques et de participants à la recherche (Arrigo, Gall, Delogne et Molin, 1994; Liptrott, Orlando, Clerici, Cocquio et Martinelli, 2009; Lubejeko et al., 2011; ONS, 2016; Showalter et al., 2017).

Des cours supplémentaires, de la formation et de l'enseignement supérieur ont été recensés comme des éléments importants répondant aux besoins de rendement et de pratique de l'IRC en oncologie (Arrigo et al., 1994). La plupart des programmes comprenaient à la fois de la formation en oncologie et en recherche clinique pour guider les nouvelles infirmières, bien que le manque de formation en oncologie soit un problème récurrent dans la littérature, problème qui aggrave les obstacles évidents liés au recrutement des infirmières non spécialisées en oncologie lorsque celles-ci sont en nombre insuffisant (Arrigo et al., 1994; Herena, Paguio et Pulone, 2018). Les infirmières formées à la fois en oncologie et en 
recherche clinique étaient apparemment plus satisfaites des connaissances acquises et ont fait état de commentaires très positifs sur leur performance par rapport à leurs collègues qui n'avaient pas eu ce type d'enseignements (Arrigo et al., 1994; Liptrott et al., 2009; Showalter et al., 2017). Malgré ces résultats positifs signalés, les IRC en oncologie estimaient que la formation pourrait être enrichie en fournissant aux apprenants plus d’outils pédagogiques (Liptrott et al., 2009).

Un problème persistant dans la littérature est le manque de normalisation dans l'éducation et la formation de l'IRC en oncologie dans les milieux de pratique. Ce manque de normalisation n'est pas limité à l'enseignement et à la formation de l'IRC en oncologie, mais s'étend également à la description du rôle, des responsabilités et des attentes envers l'IRC en oncologie. À mesure que la spécialité des soins infirmiers de recherche clinique progresse avec des définitions et des normes de pratique, on observe également une tendance constante à la normalisation par l'incorporation de mesures dans l'élaboration des programmes d'études de l'IRC en oncologie, comme on l'a vu dans le CTNQ d'Ehrenburger et de Lillington de 2004, les Clinical Trial Nurse Core Competency Guidelines de l'ONS, et l'American Nurse Association's Clinical Research Nursing Scope and Standards of Practice (ANA et IACRN, 2016; Catania et al., 2012, Herena, Paguio et Pulone, 2018).

La possession d'un diplôme d'études supérieures est une attente croissante dans un poste d'IRC en oncologie et, dans certains domaines, il est considéré comme une qualification nécessaire. Dans une étude nationale comptant 61 IRC en oncologie œuvrant dans des essais cliniques en Australie, 42 participants (69\%) avaient fait des études universitaires; d'entre eux, 9 étaient titulaires d'une maîtrise et 32 avaient un diplôme ou un certificat de troisième cycle (Scott et al., 2013). Bien que les études universitaires ne soient pas toujours requises et qu'il existe des obstacles à l'enseignement (ex. frais assumés par l'employeur, manque de congés et appui limité des employeurs pour le développement de la carrière), la plupart des IRC en oncologie participantes ont exprimé un grand intérêt pour les études aux cycles supérieurs. En tenant compte de la complexité accrue des essais cliniques sur le cancer, des compétences, des connaissances et des qualifications spécialisées exigées des infirmières de recherche en oncologie, des lacunes en matière d'enseignement et de formation et d'un parcours professionnel ambigu, Scott et collaborateurs (2013) ont suggéré que non seulement la formation universitaire comblerait ces lacunes, mais qu'elle devrait également être considérée comme une qualification de l'infirmière de recherche clinique et une ligne directrice des pratiques exemplaires.

\section{Rôle de l'IRC en oncologie : pratique avancée et possibilités}

Le thème du leadership et de la pratique avancée de l'IRC en oncologie a été recensé dans quatre articles publiés au Royaume-Uni et aux États-Unis. Bird et Kirshbaum (2005) ont analysé le rôle de l'IRC en oncologie ainsi que des modèles de pratique avancée et ont proposé un cadre potentiel de pratique avancée. Ce cadre demeure à l'état préliminaire et général, et aurait besoin d'être développé pour être plus clair, spécifique et complet. Le cadre de pratique appuie un parcours de pratique et de progression de carrière et, en retour, offre des options de carrière qui peuvent se traduire par un plan précis de développement professionnel, de recrutement et de maintien du personnel (Bird et Kirshbaum, 2005; Scott et al., 2013).

Le thème du leadership et de la pratique avancée a également été relevé dans une initiative dirigée par des IRC en oncologie en conjonction avec une restructuration du département de l'IRC en oncologie du National Cancer Institute aux États-Unis. L'Oncology Nursing Society a défini les compétences de leadership de l'IRC en oncologie comme des aptitudes qui inspirent et influencent la réalisation de l'objectif commun de recherche clinique de qualité et d'amélioration des soins dans tout le continuum du cancer (Oncology Nursing Society, 2016). On a fait preuve de leadership en instituant l'IRC en oncologie comme unité de recherche indépendante, sous l'égide de l'Office of Research Nursing, au Center for Cancer Research (CCR) du National Cancer Institute des États-Unis (Ness et Royce, 2017). Les niveaux de compétences des IRC en oncologie ont été évalués et renforcés par suite de cette restructuration.

Plusieurs études ont montré un bon degré de satisfaction lorsque des infirmières de pratique avancée, des IRC et d'autres professionnels de la santé collaboraient à la recherche (Rosenzweig, Bender et Brufsky, 2005; Winter, Lavender et Blessing, 2012). Un exemple : un essai contrôlé randomisé effectué pour étudier les effets de l'érythropoïétine sur la fatigue chez les patientes atteintes de cancer du sein métastatique, où une co-chercheuse IRC a collaboré avec un collègue médecin pour étudier l'effet du médicament sur la gestion de la fatigue (Rosenzweig, Bender et Brufsky, 2005). Autre cas : une clinique de recherche dirigée par des infirmières de pratique avancée où une IRC a collaboré à une étude visant à explorer la satisfaction de la clinique (Winter, Lavender et Blessing, 2012). Bien que ces exemples de leadership de l'IRC et leur rôle en pratique avancée soient encore peu nombreux, ils soulignent le rôle de leadership potentiel que l'IRC peut jouer pour faire avancer la recherche.

\section{Complexité de la charge de travail et évaluation}

Une étude comparant les soins infirmiers de recherche clinique en endocrinologie, cardiologie et oncologie a montré que les approches de recherche en oncologie nécessitaient plus de temps et d'efforts et s'accompagnaient d'une intensité et d'une charge de travail plus élevées (Lee et Jeong, 2018). En outre, la même étude a également recensé que les critères d'inclusion/exclusion dans les essais oncologiques sont généralement plus complexes et peuvent nécessiter une formation avancée dans les différents tests utilisés pour la classification des maladies, la confirmation de l'admissibilité des participants et la formation liée à l'étude. Un manque de ressources et d'appui augmente le risque d'épuisement professionnel de l'IRC en oncologie et, par conséquent, a un impact sur la satisfaction et le maintien dans l'emploi (Good, Lubejko, Humphries et Medders, 2013; Lee et Jeong, 2018; Milani et al., 2017). 
Les paramètres mesurant la charge de travail des IRC ont été définis dans le Wichita Community Clinical Oncology Program Protocol Acuity Tool (WPAT), le Nursing Time Required by Clinical Trial - Assessment Tool (NTC-TA) d'Italie, et la Resource-Based Relative Value Scale for Clinical Research Nurses' Workload (échelle RBRV) de Corée (Good, Lubejko, Humphries et Medders, 2013; Lee et Jeong, 2018; Milani et al., 2017). Bien que ces outils provenaient de différents pays où la conception et la réglementation du rôle et de la pratique de l'IRC en oncologie peuvent ne pas être uniformes, ces outils sont similaires en termes d'objectifs et d'indicateurs de mesure. Le service de soins infirmiers, les soins aux patients et l'acuité des soins, la phase de recherche et la complexité du protocole sont les indicateurs de chacun de ces outils. Le score obtenu indique non seulement la charge de travail individuelle de chaque infirmière, mais fournit également des données quantifiables et objectives indiquant s'il est nécessaire de recruter, d'apporter un appui à la charge de travail ou encore de redistribuer autrement le travail entre les membres du personnel.

Lévaluation du WPAT est basée sur le calcul du score d'acuité, un score de classification des patients et de classification du protocole d'étude. Les éléments pris en compte dans le calcul du score sont le statut de l'essai du patient (étude en cours) et le type d'étude (traitement versus lutte contre le cancer) (Good et al., 2013). Le score d'acuité est utilisé pour la comparaison et la redistribution de la charge de travail entre les IRC en oncologie et détermine le seuil d'augmentation du personnel. Le WPAT est utilisé dans son établissement local depuis 11 ans, et aurait une bonne cohérence et validité internes. Toutefois, il n'a pas été validé dans d'autres établissements, milieux de pratique ou pays (Good et al., 2013).

Le NTC-TA a été conçu sur la base d'un calcul des coefficients standards des activités de soins infirmiers. Pour déterminer le nombre total d'heures de travail nécessaires par étude, les coefficients standards évaluent les activités principales effectuées par l'IRC en oncologie et la complexité moyenne des phases de recherche (phases I, II et III) (Milani et al., 2017). Les activités principales des soins infirmiers ont été déterminées à partir de la revue et de l'analyse de la littérature, des avis d'experts et de la vérification des membres. Les activités ont été chronométrées et codées par un observateur externe indépendant avant d'être saisies pour le calcul du coefficient standard (Milani et al., 2017).

Enfin, le Resource-Based Relative Value Score (RBRVS) est un système de notation de la charge de travail destiné à être utilisé pour les soins infirmiers de recherche clinique auprès de populations souffrant de maladies diverses. Il a été adopté à partir d'un outil utilisé dans le calcul des honoraires des médecins, des chirurgiens et du salaire des infirmières en soins intensifs, l'activité ou le service de soins infirmiers étant l'unité de base pour le calcul de la charge de travail. Une activité infirmière menée dans plus de $98 \%$ des cas par une IRC en oncologie est sélectionnée comme référence pour évaluer d'autres activités infirmières. L'intensité de chaque activité est définie en termes d'effort technique (compétence), d'effort mental (connaissances et jugement professionnels) et de stress (hypothèse que la tension de l'énergie psychologique varierait avec l'expérience) (Lee et Jeong, 2018). Le score de charge de travail produit reflète les activités infirmières fournies ainsi que leur intensité associée.

\section{Perspectives internationales}

La mondialisation des essais cliniques a entraîné une augmentation du nombre d'essais cliniques internationaux et multisites réalisés et, par conséquent, une reconnaissance accrue de l'impact de la pratique de l'IRC en oncologie et du développement professionnel dans les essais cliniques internationaux multicentriques. Par la suite, la hausse constante du nombre d'essais internationaux a déclenché une vague d'études sur le rôle de l'IRC en oncologie dans les pays souhaitant augmenter leur participation aux essais cliniques. Le CTNQ élaboré par Ehrenberger et Lillington (2004) a été traduit et validé en italien et en coréen (Catania et al., 2012; Catania et al., 2008; Choi et Park, 2018). En Italie, le rôle de l'IRC est davantage axé sur les soins directs aux patients, alors qu'en Amérique du Nord, le rôle de l'IRC est double : soins directs aux patients et gestion des études (Catania et al., 2012). La participation des infirmières à la recherche en matière de leadership a été signalée comme étant faible, bien que la reconnaissance du potentiel de pratique avancée dans le domaine des essais cliniques en soins infirmiers soit en augmentation en Italie (Catania et al., 2012). De même, deux études coréennes ont souligné l'importance du rôle de l'IRC en oncologie, bien qu'il soit à un stade de développement précoce. L'une de ces études portait spécifiquement sur la délimitation du rôle, visant à traduire et à valider le CTNQ en coréen (Choi et Park, 2018). Une deuxième étude, principalement axée sur la mesure de la charge de travail de l'IRC comme décrit ci-dessus, a jeté un autre éclairage sur le rôle de l'IRC en oncologie en Corée, et conclu que l'intensité des activités infirmières est plus élevée en oncologie, par rapport aux essais cliniques en cardiologie et en endocrinologie (Lee et Jeong, 2018).

Une étude japonaise a fait état de conclusions sur la pratique des IRC en général par rapport à la pratique des IRC spécifiques à l'oncologie, qui en est encore à ses débuts (Matsumoto, Nagamura, Ogami, Yamashita et Kamibeppu, 2011). Létude a révélé que les soins aux patients participant à des essais cliniques sont généralement dispensés par des infirmières de chevet, tandis que les coordonnateurs de recherche clinique, dont l'expérience professionnelle peut ou non être dans le domaine des soins infirmiers, se concentrent sur la gestion et la facilitation des études. Les auteurs ont noté que l'un des obstacles inhérents à ce modèle d'administration des essais cliniques est le manque apparent de communication et de connexion entre les infirmières de services d'hospitalisation, les coordonnateurs de recherche clinique et le reste de l'équipe de recherche. En outre, en ce qui concerne les relations entre le coordonnateur de recherche clinique et les patients, le coordonnateur de recherche clinique a été décrit comme jouant le rôle de liaison de communication et de défense des droits des patients, même si l'étendue des soins directs aux patients n'est que vaguement décrite (Fujiwara et al., 2017). 
Une étude taïwanaise a identifié le premier groupe d'IRC en oncologie formé en 1989, notant que les IRC en oncologie restaient un domaine de pratique spécialisé mal intégré et peu élaboré (Cheng, Lai et Yu, 1998). En raison du manque d'intégration et du caractère embryonnaire de ce domaine spécialisé, les auteurs ont conclu que l'IRC était perçu comme un cheminement de carrière ardu, ce qui avait considérablement nui à son développement. L'IRC en oncologie pourrait bien avoir considérablement évolué par rapport à ces débuts il y a plus de vingt ans, étant donné que le pays se classe maintenant au $14^{\mathrm{e}}$ rang mondial pour la participation aux essais cliniques (WHO, 2017).

\section{DISCUSSION}

Les études sur les soins infirmiers de recherche clinique menées au cours des quatre dernières décennies indiquent une transformation de la pratique en une spécialité qui est maintenant reconnue par l'American Nurses Association (ANA et IACRN, 2016). En outre, tout au long de l'émergence et de la transformation des soins infirmiers de recherche clinique en un domaine de pratique spécialisé, l'oncologie a été en tête pour le nombre d'essais de soins de santé et le développement de la pratique de l'IRC en oncologie, par rapport à d'autres types de maladies. Depuis les premières descriptions anecdotiques, le rôle de l'IRC en oncologie a rapidement évolué et continue actuellement à être étayé par des recherches empiriques et des données pratiques. Non seulement le rôle a été étendu à un champ d'application complet, mais il est maintenant considéré comme une pratique avancée.

Les thèmes recensés dans cette étude portent à croire que la prochaine transformation de l'IRC en oncologie sera de devenir un domaine de pratique sous-spécialisé, distinct des soins infirmiers généralistes en recherche clinique. À bien des égards, l'appel de Peplau il y a presque quinze ans semble se concrétiser (1965/2003), à savoir que :

«... la spécialisation au sein d'une profession tend à être une division du domaine générique [...] elle part de travailleurs pionniers qui voient ou sentent un grand besoin de prendre une direction particulière et poussée [...] [après quoi] les travailleurs typiques s'exprimant au nom et par l'intermédiaire de l'organisation professionnelle décident de l'orientation en matière de spécialisation qui sera développée ou révisée. " (p. 3-4) [Traduction]

En ce qui concerne cette spécialisation des soins infirmiers professionnels, Peplau (1965/2003) a en outre avancé qu'un apport de connaissances est l'un des antécédents de la spécialisation. Avec l'augmentation des nouvelles connaissances, qu'il s'agisse de sciences fondamentales/appliquées ou de progrès technologiques, les praticiens sont tenus de développer des compétences technologiques et intellectuelles complexes (Peplau, 1965/2003). Par conséquent, les progrès scientifiques et technologiques contribuant à une activité accrue de la recherche sur le cancer, on s'attend à une hausse subséquente du nombre et de la complexité des essais cliniques et à ce qu'ils dictent non seulement la quantité d'IRC en oncologie, mais aussi la qualité de ces IRC.
La nature dynamique de la biologie et de la génétique du cancer rend le diagnostic, la gestion et les soins du cancer de plus en plus complexes et mouvants. En outre, les patients cancéreux ont des besoins particuliers exigeant que les infirmières qui soignent ces patients aient des connaissances et des compétences spécialisées en oncologie. Sur le plan clinique, ces complexités biomédicales, traitements et divers besoins des patients cancéreux exigent des connaissances infirmières avancées sur la maladie sous-jacente et les effets secondaires des traitements, afin de protéger les patients et d'assurer leur bien-être au cours du processus. En bref, c'est là que résident l'ironie des essais cliniques en oncologie et l'importance de la spécialisation de l'IRC en oncologie, à savoir que les patients qui reçoivent de nouveaux traitements susceptibles de prolonger leur vie sont ceux qui sont également soumis à des niveaux de risque et d'incertitude plus élevés en ce qui concerne l'impact et l'efficacité thérapeutiques. Lorsque l'on considère la complexité accrue de la sécurité des sujets d'étude et du consentement éclairé, en plus des tâches délicates à effectuer auprès de groupes vulnérables comme les enfants, les personnes âgées et les minorités ethniques, les connaissances et les compétences avancées de l'IRC en oncologie deviennent d'autant plus évidentes.

Les conclusions de cette revue indiquent que la préparation et la formation en oncologie et en recherche clinique sont essentielles non seulement pour la sécurité des participants à l'étude, mais aussi en termes de rendement, de confiance et de satisfaction de l'IRC en oncologie. Jusqu'à présent, les interventions de l'Oncology Nurse Society ont joué un rôle important dans l'avancement de l'IRC en oncologie le long du parcours de sous-spécialité, notamment en produisant des lignes directrices essentielles qui ont façonné la pratique infirmière de recherche clinique en oncologie. Parallèlement, les travaux de l'ONS sur les compétences et les lignes directrices de l'IRC en oncologie ont permis de distinguer les soins infirmiers en recherche clinique en oncologie de l'infirmière de recherche clinique en général. Ce caractère distinct, avec le cadre de la pratique de l'IRC en oncologie de Purdom et collaborateurs, signifie que l'IRC en oncologie est de plus en plus reconnue comme une sous-spécialité des soins infirmiers en oncologie.

Dans cette étude, les thèmes recensés tendent à indiquer un manque de normalisation, notamment en ce qui concerne les exigences en matière de formation et de qualification de l'IRC en oncologie. Comme moyen d'assurer la qualité et la responsabilité, la certification spécialisée d'IRC en oncologie est requise. Définir les compétences et décrire précisément le rôle de l'IRC en oncologie semblent être les prochaines étapes logiques de ce processus, étapes qui pourraient être facilement intégrées dans les pratiques et les politiques des établissements locaux, si elles ne sont pas normalisées dans tous les milieux de pratique.

Étant donné que la formation en recherche clinique et l'expérience en oncologie sont toutes deux cruciales pour l'implantation réussie du rôle d'IRC en oncologie, l'intégration de ces exigences à la formation actuelle semble disparate et sous-développée; il faudrait incorporer le travail d'IRC au 
programme de soins infirmiers en oncologie. De même, il est nécessaire de poursuivre la discussion sur l'enseignement supérieur et la préparation à la maîtrise pour les IRC en oncologie. Cela permettrait de reconnaître le caractère avancé du rôle et d'offrir un parcours de carrière clair, et aurait des répercussions positives sur le recrutement et le maintien en poste.

L'exploration des titres de pratiques avancées pour le rôle de l'IRC en oncologie est à ses débuts. Bien que les travaux de Bird et Kirshbaum (2006) aient amorcé un dialogue important et défini le fondement théorique de la pratique avancée de l'infirmière de recherche en oncologie, la poursuite du développement du cadre de la pratique et des concepts dans un rôle de pratique avancée doit avoir lieu dès la publication. Le rôle des infirmières dans les centres de recherches cliniques, des infirmières co-chercheuses en collaboration avec un oncologue, et d'autres modèles de leadership favorisent des modèles de pratiques avancées qui pourraient être adoptées dans une plus large mesure par l'IRC en oncologie. Cela dit, il est encore prématuré d'affirmer une désignation de pratique avancée ou un cheminement de carrière clair pour l'infirmière de recherche clinique en oncologie sans recherches supplémentaires. Néanmoins, le récent cadre élaboré par l'enquête transversale de Purdom et collaborateurs (2017) sur l'IRC en oncologie représente un premier pas important vers la normalisation de la pratique et le perfectionnement professionnel.

Sur les 29 études incluses dans cet examen, deux seulement portaient sur des IRC canadiennes : l'une partiellement seulement (échantillon mixte d'IRC provenant des États-Unis et du Canada) (Cooperative Group of Clinical Trials), et l'autre avec un échantillon exclusivement canadien. Ce peu de recherche en contexte canadien est étonnant puisque le Canada se classe septième au monde pour le nombre d'essais cliniques (OMS, 2017). Une discordance entre la participation aux essais cliniques et les études sur le rôle de l'infirmière de recherche a également été recensée à Taïwan, où la recherche et les lignes directrices sur l'IRC sont sous-développées par rapport à la forte participation du pays à la réalisation d'essais cliniques. Par ailleurs, les publications sur les pratiques de l'IRC au Canada ont été largement limitées à des discussions anecdotiques et à des articles d'opinion, révélant une lacune considérable de la recherche qui nécessiterait une attention accrue. Alors que l'IRC en oncologie, dans le domaine de l'oncologie et ailleurs, a commencé à se positionner comme sous-spécialité unique, l'évolution est entravée par le manque de recherche pour justifier les affirmations de la sous-spécialité et pour développer des directives basées sur des données probantes.

Du point de vue du développement de la pratique avancée de l'IRC en oncologie, des recherches supplémentaires sont nécessaires pour mieux définir les aspects de la pratique avancée et délimiter les rôles. Compte tenu du milieu de recherche clinique et de ses compétences en matière de recherche, il est opportun et à-propos pour l'IRC en oncologie de mener des recherches sur l'avancement des essais cliniques infirmiers. La recherche, les publications et la diffusion non seulement établissent la base de données probantes pour la pratique avancée de l'IRC en oncologie, mais fournissent également des preuves pour garantir que les soins infirmiers sont reconnus au même titre que les autres professions participant à l'activité de recherche. La question est particulièrement urgente si l'on considère que la recherche en soins infirmiers oncologiques dans les essais cliniques a été essentiellement descriptive, lacunaire tant sur le plan de la qualité et que de la quantité (Molassiotis et al., 2006).

À l'échelle internationale, là où la recherche en matière de soins infirmiers dans les essais cliniques semble être la plus avancée, c'est dans les pays développés où les systèmes de soins de santé, le financement de la santé et les professions infirmières sont bien établis. En raison du taux actuel de croissance économique dans de nombreux pays en développement, de l'augmentation de la prévalence du cancer à l'échelle mondiale et du mouvement en faveur des essais cliniques multicentriques sur le cancer, le rôle des soins infirmiers en oncologie et dans les essais cliniques devra se développer à un rythme similaire. Afin de faciliter cette évolution et de faire progresser l'IRC en oncologie vers les pays à faibles ressources, des normes internationales d'IRC en oncologie sont nécessaires.

La participation des infirmières et les bourses d'études au sein des établissements de recherche clinique peuvent être présentes localement et dans le contexte de leur propre environnement de pratique ou s'étendre à des collaborations et partenariats à l'étranger. L'appui à la profession, de la part des gouvernements ou des établissements de recherche locale est essentiel pour développer ce rôle de pratique infirmière avancée. Tout aussi important, l'IRC en oncologie doit elle-même jouer un rôle crucial en plaidant pour le développement de la pratique avancée de l'IRC en oncologie, car elle est à l'épicentre de la recherche sur les essais cliniques et possède des connaissances d'initiés sur l'importance, les obstacles et la complexité de ce travail.

\section{Limites}

Cette revue n'est pas dépourvue de limites. Comme elle s'est limitée aux études en anglais, les répercussions globales de l'IRC en oncologie n'ont peut-être pas été entièrement prises en compte. Une autre limite est que la sélection et l'extraction des données ont été effectuées par une seule personne, car l'étude était un projet de recherche de maîtrise; cependant, le processus de décision a été guidé et examiné par la personne la supervisant, un bibliothécaire de recherche principal, et d'autres membres du comité de diplômés. Cette limite a été aussi atténuée par la conservation d'une documentation complète sur les processus de l'étude et par les décisions prises lors de la collecte et de la communication des résultats, qui sont ouvertes à l'examen des lecteurs, renforçant la transparence de l'étude (Arksey et O’Malley, 2005; Armstrong et al., 2011).

\section{CONCLUSION}

L'innovation en matière de soins et de traitements contre le cancer évolue à un rythme remarquable depuis soixantedix ans. Au sein de l'équipe de recherche, l'IRC en oncologie joue un rôle essentiel dans l'avancement scientifique des nouveaux traitements contre le cancer, qui permettent aux patients de vivre plus longtemps avec une meilleure qualité de vie. Les 
soins aux patients, la protection de leurs droits, leur sécurité et leur bien-être sont au cœur de la recherche clinique, et en préservant ces qualités selon les normes les plus élevées, l'IRC en oncologie facilite le fonctionnement et la réussite de la recherche clinique. Malgré les progrès rapides réalisés grâce à la recherche sur les essais cliniques en oncologie, le rôle spécialisé de l'infirmière de recherche clinique en oncologie n'a pas fait l'objet d'une étude systématique, un état de fait qui entrave le développement d'une approche normalisée du rôle

\section{RÉFÉRENCES}

American Nurses Association and International Association of Clinical Research Nurses. (2016). Clinical Research Nursing: Scope and Standards of Practice.

Allison, M. (2012). Reinventing clinical trials. Nature biotechnology, $30(1), 41$.

Arksey, H., \& O'Malley, L. (2005). Scoping studies: Towards a methodological framework. International Journal of Social Research Methodology, 8(1), 19-32.

Armstrong, R., Hall, B. J., Doyle, J., \& Waters, E. (2011). 'Scoping the scope' of a Cochrane review. Journal of Public Health, 33(1), 147-150.

Arrigo, C., Gall, H., Delogne, A., \& Molin, C. (1994). The involvement of nurses in clinical trials: results of the EORTC Oncology Nurses Study Group survey... European Organization for Research and Treatment of Cancer. Cancer Nursing, 17(5), 429-433.

Bevans, M., Hastings, C., Wehrlen, L., Cusack, G., Matlock, A. M., Miller-Davis, C., Tondreau, L., Walsh, D., \& Wallen, G. R. (2011). Defining clinical research nursing practice: Results of a role delineation study. Clinical and Translational Science, 4(6), 421-427.

Bird, J., \& Kirshbaum, M. (2005). Towards a framework of advanced nursing practice for the clinical research nurse in cancer care. Clinical Effectiveness in Nursing, 9(3-4), 161-171. https://doi. org/10.1016/j.cein.2006.08.008.

Carlson, C., Reilly, M., \& Hitchens, A. (2005). An innovative approach to the care of patients on phase I and phase II clinical trials: The role of the experimental therapeutics nurse. Journal of Pediatric Oncology Nursing, 22(6), 353-364. https://doi. org/10.1177/1043454205281763.

Castro, K., Bevans, M., Miller-Davis, C., Cusack, G., Loscalzo, F., Matlock, A. M., \& Hastings, C. (2011). Validating the clinical research nursing domain of practice. Oncology Nursing Forum. 8(2). https://doi.org/10.1188/11.ONF.E72-E80.

Catania, G., Poirè, I., Bernardi, M., Bono, L., Cardinale, F., \& Dozin, B. (2012). The role of the clinical trial nurse in Italy. European Journal of Oncology Nursing, 16(1), 87-93. https://doi.org/10.1016/j. ejon.2011.04.001.

Catania, G., Poirè, I., Dozin, B., Bernardi, M., \& Boni, L. (2008). Validating a measure to delineate the clinical trials nursing role in Italy. Cancer Nursing, 31(5), E11-15.

Chang, A. (2008). An exploratory survey of nurses' perceptions of phase I clinical trials in pediatric oncology. Journal of Pediatric Oncology Nursing, 25(1), 14-23.

Cheng, A.-L., Lai, Y.-H., \& Yu, Y.-C. (1998). Reserach nurses for clinical trials of anti- cancer drugs: developing a new subspecialty in Taiwan. Drug Information Journal, 32, 1279S-1281S.

Choi, I. J., \& Park, H.-J. (2018). Validation of a questionnaire to delineate the clinical trial nursing roles in Korea. Cancer Nursing, 41(3), E30-E37.

Coulson, S., \& Phelan, L. (2000). Clinical research in paediatric oncology and the role of the research nurse in the UK. European Journal of Oncology Nursing, 4(3), 154-161. https://doi.org/10.1054/ ejon.2000.0100. de ces soins infirmiers spécialisés. Cette revue exploratoire fait la synthèse de l'évolution du domaine spécialisé des soins infirmiers oncologiques et des soins infirmiers dans les essais cliniques, décrit la situation actuelle dans les écrits, recense les lacunes dans les connaissances et émet des recommandations pour les recherches et orientations futures.

\section{CONFLIT D'INTÉRÊTS}

Aucun conflit d'intérêts à signaler.

CRN 2010 Domain of Practice Committee. (2009). Building the foundation for clinical research nursing: Domain of practice for the specialty of clinical research nursing. National Institutes of Health Clinical Center, Nursing and Patient Care Services. Available at http://www.cc.nih.gov/nursing/crn/DOP_document.pdf

Ehrenberger, H. E., \& Lillington, L. (2004). Development of a measure to delineate the clinical trials nursing role. Oncology Nursing Forum, 31(3), E64-68.

Fujiwara, N., Ochiai, R., Shirai, Y., Saito, Y., Nagamura, F., Iwase, S., \& Kazuma, K. (2017). Qualitative analysis of clinical research coordinators' role in phase I cancer clinical trials. Contemporary Clinical Trials Communications, 8, 156-161.

Good, M. J., Lubejko, B., Humphries, K., \& Medders, A. (2013). Measuring clinical trial-associated workload in a community clinical oncology program. Journal of Oncology Practice, 9(4), 211215. https://doi.org/10.1200/JOP.2012.000797.

Hastings, C. E., Fisher, C. A., McCabe, M. A., \& Consortium, N. C. R. N. (2012). Clinical research nursing: a critical resource in the national research enterprise. Nursing Outlook, 60(3), 149-156. e143.

Herena, P., Paguio, G., \& Pulone, B. (2018). Clinical research nurse education: using scope and standards of practice to improve care. Clinical Journal of Oncology Nursing, 22(4), 450-452.

Hubbard, S. M., \& Donehower, M. G. (1980). The nurse in a cancer research setting. In Seminars in Oncology Nursing, 7(1).

Jenkins, J., \& Hubbard, S. (1991). History of clinical trials. In Seminars in Oncology Nursing, 7(4).

Lee, S., \& Jeong, I. S. (2018). A resource-based relative value for clinical research nurses' workload. Therapeutic Innovation and Regulatory Science, 52(3), 313-320.

Lefebvre, C., Glanville, J., Briscoe, S., Littlewood, A., Marshall, C., Metzendorf, M.-I., Noel-Storr, A., Rader, T., Shokraneh, F., Thomas, J., Wieland, L. S. Chapter 4: Searching for and selecting studies. In: Higgins, J. P. T., Thomas, J., Chandler, J., Cumpston, M., Li, T., Page, M. J., Welch, V. A. (editors). Cochrane Handbook for Systematic Reviews of Interventions version 6.2 (updated February 2021). Cochrane, 2021. Available from www.training.cochrane.org/ handbook

Levac, D., Colquhoun, H., \& O’Brien, K. K. (2010). Scoping studies: advancing the methodology. Implementation Science, 5(1), 69.

Liptrott, S., Orlando, L., Clerici, M., Cocquio, A., \& Martinelli, G. (2009). A competency based educational programme for research nurses: An Italian experience. ecancermedicalscience, 3(1). https:// doi.org/10.3332/ecancer.2009.134

Loh, W. Y., Butow, P. N., Brown, R. F. \& Boyle, F. (2002). Ethical communication in clinical trials: issues faced by data managers in obtaining informed consent. Cancer, 95(11), 2414-21. https://doi. org/10.1002/cncr.10994

Lubejko, B., Good, M., Weiss, P., Schmieder, L., Leos, D., \& Daugherty, P. (2011). Oncology Clinical Trials Nursing. Clinical Journal of Oncology Nursing, 15(6). 
Matsumoto, K., Nagamura, F., Ogami, Y., Yamashita, N., \& Kamibeppu, K. (2011). Difficulties of nursing staff involved in phase 1 oncology trials in Japan. Cancer Nursing, 34(5), 369-375.

McEvoy, M. D., Cannon, L., \& MacDermott, M. L. (1991). The professional role for nurses in clinical trials. Seminars in Oncology Nursing, 7(4), 268-274.

Medical Research Council Investigation. (1948). Streptomycin treatment of tuberculosis. British Medicine Journal, 2, 769-782. https://doi.org/10.1136/bmj.2.4582.769

Milani, A., Mazzocco, K., Stucchi, S., Magon, G., Pravettoni, G., Passoni, C., Ciccarelli, C., Tonali, A., Profeta, T., \& Saiani, L. (2017). How many research nurses for how many clinical trials in an oncology setting? Definition of the Nursing Time Required by Clinical Trial-Assessment Tool (NTRCT-AT). International Journal of Nursing Practice, 23(1).

Molassiotis, A., Gibson, F., Kelly, D., Richardson, A., Dabbour, R., Ahmad, A. M., \& Kearney, N. (2006). A systematic review of worldwide cancer nursing research: 1994 to 2003. Cancer Nursing, 29(6), 431-440.

Mueller, M. R. (2001). From delegation to specialization: nurses and clinical trial co-ordination. Nursing Inquiry, 8(3), 182-190.

Nagel, K., Gender, J., \& Bonner, A. (2010). Delineating the role of a cohort of clinical research nurses in a pediatric cooperative clinical trials group. [Reprint in ONS Connect. 2010 May;25(5):12-3; PMID: 20486615]. Oncology Nursing Forum, 37(3), E180-185.

Ness, E. A., \& Royce, C. (2017). Clinical trials and the role of the oncology clinical trials nurse. Nursing Clinics of North America, 52(1), 133-148.

Ocker, B. M., \& Plank, D. M. P. (2000). The research nurse role in a clinicbased oncology research setting. Cancer Nursing, 23(4), 286-294.

Oncology Nurse Society. (2010). 2010 Oncology clinical trials nurse competencies. Oncology Nurse Society.
Oncology Nurse Society. (2016). 2016 Oncology clinical trials nurse competencies. Oncology Nurse Society.

Peplau, H. (2003). Specialization in professional nursing. Clinical Nurse Specialist, 17(1), 3-9.

Purdom, M. A., Petersen, S., \& Haas, B. K. (2017). Results of an oncology clinical trial nurse role delineation study. Oncology Nursing Forum, 44(5), 589-595. https://doi.org/10.1188/17. ONF.589-595

Rosenzweig, M. Q., Bender, C. M., \& Brufsky, A. M. (2005). The nurse as principal investigator in a pharmaceutically sponsored drug trial: Considerations and challenges. Oncology Nursing Forum, 32(2), 293-299.

Scott, K., White, K., Johnson, C., \& Roydhouse, J. K. (2012). Knowledge and skills of cancer clinical trials nurses in Australia. Journal of Advanced Nursing, 68(5), 1111-1121.

Scott, K., White, K., \& Roydhouse, J. K. (2013). Advancing the educational and career pathway for clinical trials nurses. Journal of Continuing Education in Nursing, 44(4), 165-170.

Showalter, B. L., Cline, D., Yungclas, J., La Frentz, K., Stafford, S. R., \& Maresh, K. J. (2017). Clinical research nursing: Development of a residency program. Clinical Journal of Oncology Nursing, 21(5), 633-636. https://doi.org/10.1188/17.CJON.633-636.

Thiers, F. A., Sinskey, A. J., \& Berndt, E. R. (2008). Trends in the globalization of clinical trials. In: Nature Publishing Group.

Winter, H., Lavender, V., \& Blesing, C. (2012). Assessing satisfaction with a nurse-led clinical trials clinic. Cancer Nursing Practice (through 2013), 11(9), 23.

World Health Organization's International Clinical Trials Registry Platform (2017). http://apps.who.int 